\title{
Acute hemorrhagic necrotizing enteritis: a case report and review of the literature
}

\author{
Shaoxiong Zeng ${ }^{1 \wedge}$, Christ-Jonathan Tsia Hin Fong ${ }^{1 \wedge}$, Leijia Li $^{1 \wedge}$, Yanping Liang ${ }^{1} \wedge$, Qiong Liang ${ }^{2}$, \\ Bo $\mathrm{Wei}^{3} \wedge, \operatorname{Jin}^{\mathrm{Ta}}{ }^{1 \wedge}$ \\ ${ }^{1}$ Department of Gastroenterology, The Third Affiliated Hospital of Sun Yat-sen University, Guangzhou, China; ${ }^{2}$ Department of Pathology, The \\ Third Affiliated Hospital of Sun Yat-sen University, Guangzhou, China; ${ }^{3}$ Department of Gastrointestinal Surgery, The Third Affiliated Hospital of \\ Sun Yat-sen University, Guangzhou, China \\ Correspondence to: Jin Tao, MD, PhD. Professor, Department of Gastroenterology, The Third Affiliated Hospital of Sun Yat-sen University, No. 600 \\ Tianhe Road, Guangzhou, China. Email: taojin3@mail.sysu.edu.cn.
}

\begin{abstract}
Acute hemorrhagic necrotizing enteritis (AHNE) is a potentially fatal infection, triggered by beta toxin produced by Clostridium perfringens type $\mathrm{C}$ and characterized by extensive hemorrhagic, inflammatory, or ischemic necrosis that mainly affects the small bowel, clinically presenting as diarrhea, hematochezia, abdominal pain and hypotensive shock. AHNE is rarely reported in humans nowadays, we present a case of AHNE in a 51-year-old man presenting as watery diarrhea, hematochezia and abdominal pain along with shortness of breath who unfortunately died of the disease despite active medical treatment and multiple surgical interventions. We aim to improve awareness of clinicians on this fulminant disease, associated with high mortality rates. This is the first case report that attempts to summarize the pathogenesis, clinical characteristics, diagnostic methods, treatment and prognosis of AHNE based on the current English literature. AHNE, which is exceedingly rare in clinical practice, has been associated with poorly specific clinical manifestations, high rates of misdiagnosis in its early stages and mortality rates in severe cases. In patients with a history of ingesting contaminated food and presenting with sudden progressively worsening abdominal pain, diarrhea, hematochezia, accompanied by hypotensive shock or ileus, AHNE should be highly suspected. In order to reduce the mortality of this disease, emphasis should be laid on early recognition and timely surgical intervention in AHNE. In severe cases, death cannot be avoided despite adopting active supportive treatment and timely surgical intervention.
\end{abstract}

Keywords: Acute hemorrhagic necrotizing enteritis (AHNE); Clostridium perfringens type C; beta toxin; case report; review

Submitted May 18, 2020. Accepted for publication Aug 21, 2020.

doi: 10.21037/apm-20-1131

View this article at: http://dx.doi.org/10.21037/apm-20-1131

\section{Introduction}

Acute hemorrhagic necrotizing enteritis (AHNE) is characterized by extensive hemorrhagic, inflammatory, or ischemic necrosis that mainly affects the small bowel, mainly presenting as diarrhea, hematochezia, abdominal pain, abdominal distention, nausea, vomiting and hypotensive shock. It was first reported in Northern Germany after World War II among chronically starved persons who ingested poorly cooked meat and was called "Darmbrand" which means "burnt bowels" $(1,2)$. A similar condition was subsequently described in Papua New Guinea among

^ ORCID: Shaoxiong Zeng, 0000-0002-7358-986X; Christ-Jonathan Tsia Hin Fong, 0000-0002-0489-342X; Leijia Li, 0000-0001-76683491; Yanping Liang, 0000-0001-5291-8479; Qiong Liang, 0000-0001-9567-9121; Bo Wei, 0000-0002-1058-5888; Jin Tao, 0000-00024027-0852. 
children and young adults after consumption of huge quantities of sweet potatoes and inadequately cooked pork contaminated with pig intestines in 1961, termed "pigbel", a pidgin English term for "abdominal pain after a pig feast" $(3,4)$. Thankfully, with the improvement in global socioeconomic conditions and general hygiene, there has been a notable decline in worldwide incidence of AHNE. It is noteworthy that AHNE which is exceedingly rare in clinical practice since 2000 , has been associated with a high rate of misdiagnosis in its early stages and high mortality rates in severe cases. In this paper, we present a case of AHNE in a 51-year-old male patient with diarrhea, abdominal pain, hematochezia and shortness of breath and review the current literature, focussing on the pathogenesis, clinical features, diagnosis, treatment and prognosis. Because of its rarity in modern life and hence its high likelihood of being underdiagnosed, we aim to raise awareness of modern clinicians on this fulminant disease. To our knowledge, this is the first case report that attempts to summarize the pathogenesis, clinical characteristics, diagnostic methods, treatment and prognosis of AHNE based on the current literature. We present the following article in accordance with the CARE reporting checklist (available at http:// dx.doi.org/10.21037/apm-20-1131).

\section{Case presentation}

On October 16, 2018, a 51-year-old man was transferred to the Third Affiliated Hospital of Sun Yat-sen University, complaining of watery diarrhea and bloody stools for the past 12 hours and abdominal pain along with shortness of breath for the past 4 hours. Prior to the onset of symptoms, the patient consumed large quantities of suspected contaminated food. Twelve hours before admission, the patient began to develop watery diarrhea alternating with bloody stools, with a total volume of about $500 \mathrm{~mL}$, accompanied by dizziness and vomiting. Eight hours later, the patient developed abdominal pain, accompanied by shortness of breath, pallor and sweating with no complaints of fever and chills.

The patient had a previous medical history of hypertension and gout over ten years but was poorly compliant to medical treatment. His surgical history was nonsignificant. He had a twenty pack-year history of drinking and denied any other specific personal or family history of other diseases.

Physical examination upon admission revealed a temperature of $36^{\circ} \mathrm{C}$, tachycardia at $102 \mathrm{bpm}$, tachypnea at
40 breaths per minute and hypotensive shock with blood pressure at $59 / 38 \mathrm{mmHg}$. The patient was somnolent and poorly responsive. Both lungs were covered with rales and phlegm sounds on auscultation. The abdomen was distended and slight tensed, tenderness and rebound tenderness were not elicited due to comatose state of the patient. No other pathological signs were noted.

Laboratory tests showed leukocytosis $\left(17.72 \times 10 \mathrm{E}^{9}\right.$ cells/L) with predominantly neutrophils $(94 \%)$, mild anemia $(94 \mathrm{~g} / \mathrm{L})$ and a normal platelet count $\left(173 \times 10 \mathrm{E}^{9}\right.$ cells/L). Liver and renal function tests showed elevated transaminases (ALT 1,999 U/L and AST 3,099 U/L), serum creatinine $(213 \mu \mathrm{mol} / \mathrm{L})$, serum uric acid $(503 \mu \mathrm{mol} / \mathrm{L})$, serum potassium $(6.65 \mathrm{mmol} / \mathrm{L})$ and a decreased albumin $(26.9 \mathrm{~g} / \mathrm{L})$. Serum C-reactive protein and procalcitonin were increased at $554 \mathrm{mg} / \mathrm{L}$ and greater than $100 \mathrm{ng} / \mathrm{mL}$ respectively. Arterial-blood gas (ABG) analysis revealed acidosis ( $\mathrm{pH} 7.2)$, normocapnia $\left(\mathrm{pCO}_{2} 32.8 \mathrm{mmHg}\right)$ and hypoxemia $\left(\mathrm{pO}_{2} 29.9 \mathrm{mmHg}\right)$ with hyperlactemia (lactate concentration $13.5 \mathrm{mmol} / \mathrm{L}$ ). On admission, a Chest computed tomography (CT) scan revealed bilateral pneumonia with consolidation in the upper lobe of the right lung (Figure 1A). The abdominal CT scan revealed diffuse peritonitis and marked edema with thickening of the small intestinal walls (Figure 1B,C).

The patient who presented with respiratory failure, hyperkalemia and septic shock, was immediately started on mechanical ventilation, continuous renal replacement therapy, aggressive fluid resuscitation with vasopressors dopamine and norepinephrine to maintain adequate mean arterial pressure (MAP) and drotaverine to relieve gastrointestinal cramps. Initial diagnosis of severe pneumonia, multiple organ dysfunction syndrome and acute severe gastroenteritis was based on the clinical findings including patient history, symptoms and work-up findings. Empiric intravenous antibiotic therapy with meropenem $2 \mathrm{~g}$ TID, tigecycline $100 \mathrm{mg}$ BID and moxifloxacin $0.4 \mathrm{~g}$ QD was administered. Hydrocortisone $0.1 \mathrm{~g}$ TID was injected to reduce the inflammatory response. In addition, high-dose gamma globulin was given to boost immunity along with human serum albumin, hepatoprotective treatment and nutrition support. On October 24, 2018, we initiated ventilator weaning protocols when inflammatory indices and ABG values of the patient significantly improved and bedside chest $\mathrm{X}$-rays showed significant improvement (Figure 1D). On October 24, 2018 diarrhea recurred with a daily volume of 500-1,000 mL. Sputum and blood cultures were negative, but urine and stool cultures were positive 

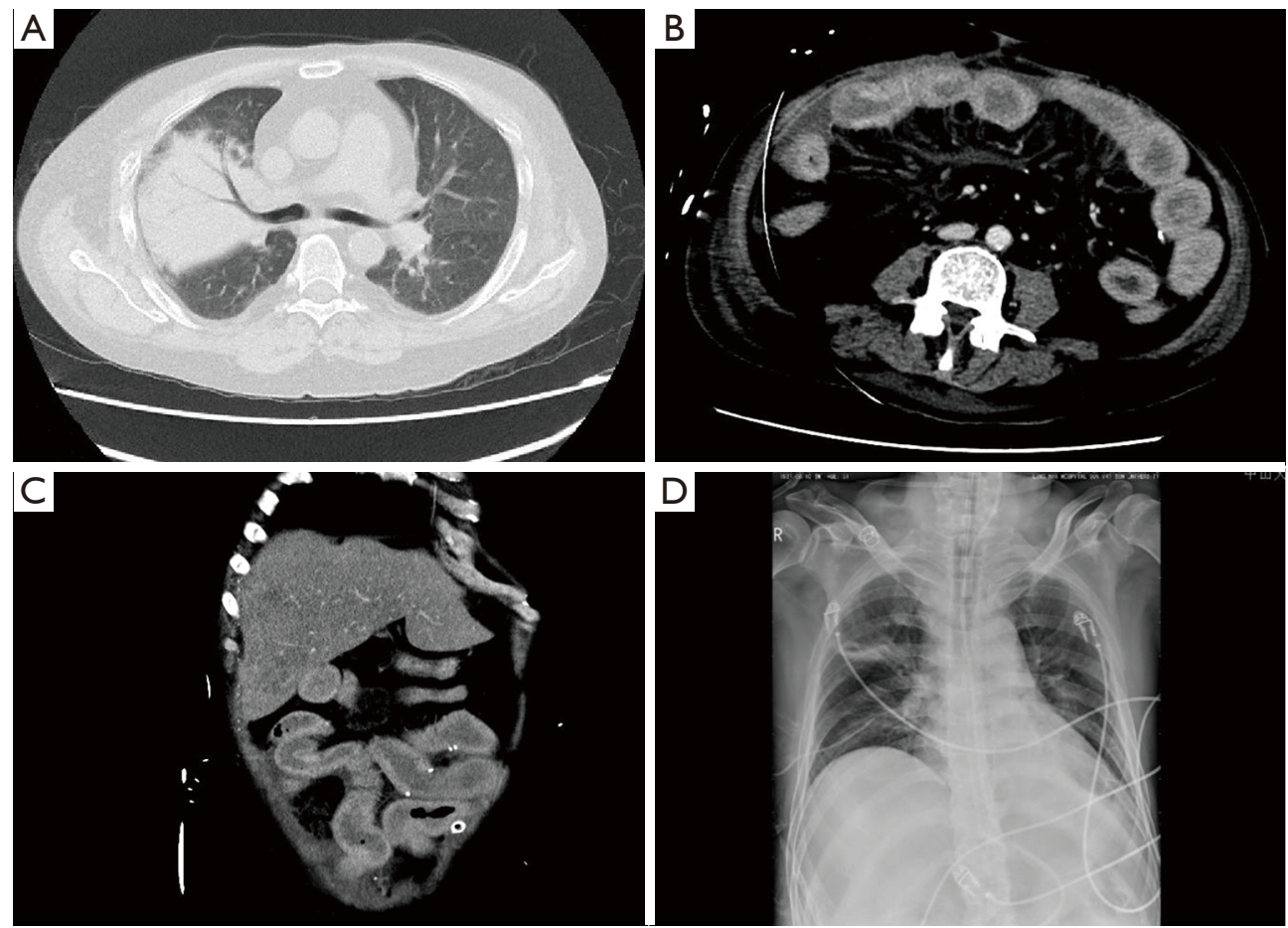

Figure 1 Computed tomography (CT) imaging of the patient. (A) Chest computed tomography (CT) scan showed bilateral pneumonia with consolidation in upper lobe of the right lung and air bronchogram sign; (B) contrast-enhanced abdominal CT scan showed diffuse peritonitis and marked edema of the small intestine with significant enhancement of the intestinal wall; (C) coronal reconstruction of abdominal CT showed thickening and edema of the intestine; (D) bedside chest radiography showed significant improvement of the upper lobe of the right lung.

for Candida albicans. Intravenous antifungal therapy with caspofungin $500 \mathrm{mg}$ QD was administered. On October 27, 2018, gastrointestinal hemorrhage recurred as hematochezia and hematemesis, with a total volume of 2,000 mL. After failure of conservative treatment, a laparotomy was finally performed revealing multisegmental bowel necrosis from the proximal jejunum to the distal ileum with severe congestion and dark purple appearance and multiple mesenteric ulcer and abscesses, rupture of the small intestine $70 \mathrm{~cm}$ proximal to the ileocecum. The necrotic bowel was then resected (Figure 2). Histopathological examination of the resected bowel showed extensive inflammation and necrosis of the mucosa, partial intestinal wall full-thickness necrosis, subserosal hemorrhage and fibrinoid vascular necrosis accompanied by endovascular thrombosis, with large-scale local infiltration of neutrophils, suggestive of AHNE (Figure 3). The final diagnosis of the presented case was AHNE. On November 2, 2018, the patient developed massive gastrointestinal bleeding (about 3,000 $\mathrm{mL}$ ), prompting a second emergency laparotomy for hemostasis. The patient postoperatively developed necrosis at the incision site which complicated with infection (Figure 2D). He unfortunately died of the disease on December 3, 2018 following repeated bouts of hemorrhage and infection despite active medical treatment and multiple surgical interventions (for details of the course, see Figure 4). All procedures performed in studies involving human participants were in accordance with the ethical standards of the institutional and/or national research committee(s) and with the Helsinki Declaration (as revised in 2013). The patient and his legal guardian provided informed written consent for publication of this study and any accompanying images during the treatment.

\section{Discussion}

AHNE is an acute, life-threatening disease characterized by extensive segmental hemorrhagic, inflammatory, or ischemic necrosis that mainly affects the small bowel and occasionally the large intestine. AHNE is rare and 

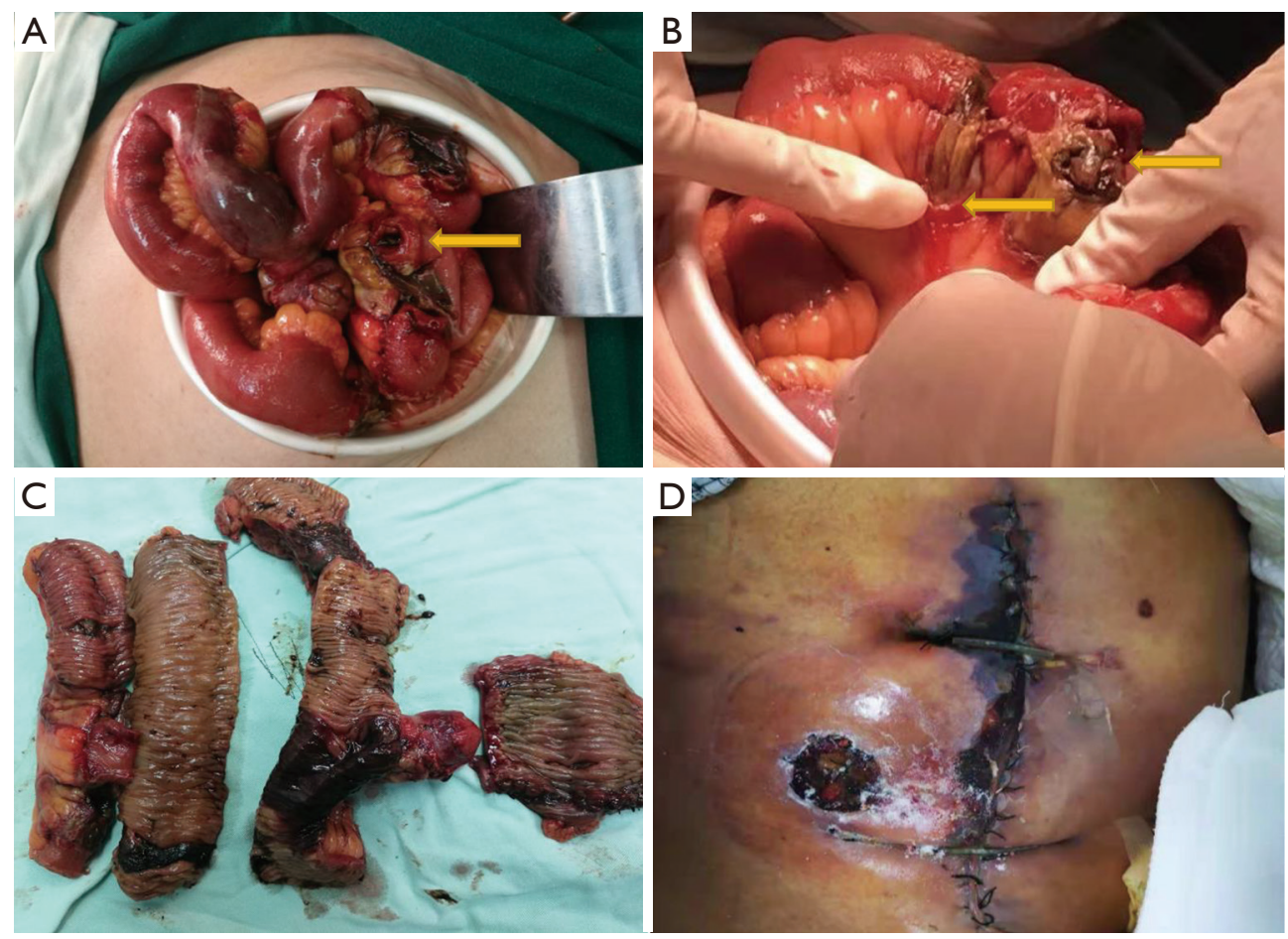

Figure 2 Operative picture of the patient. (A) Laparotomy revealed multisegmental bowel necrosis from the proximal jejunum to the distal ileum with severe congestion, edema and dark purple appearance; rupture of the small intestine $70 \mathrm{~cm}$ proximal to the ileocecum was visible (see the arrow); (B) multiple mesenteric ulcer and abscesses were visible (see the arrow); (C) the lesions were segmentally distributed and involved the serosa; transmural necrosis and hemorrhage were visible; (D) post-operative incision site necrosis complicated with infection.

occurs sporadically in developed countries but has higher prevalence in Southeast Asia, for instance in Papua, New Guinea, where interestingly, it has been reported to be the most common cause of death in children $(4,5)$. We report a case of AHNE in a 51-year-old male patient with diarrhea, hematochezia, abdominal pain and shortness of breath and reviewed the current literature. The demographics and clinical characteristics of the 10 cases are summarized in Table 1. From the review, we can establish that the age of the patients ranged from 12 to 66 years with a median age of 47 years inclined towards middle-aged adults while the male:female ratio was 5:5. The small bowel was involved in nine cases and the colon in two cases, suggesting that small bowel is the main affected organ. Five patients died of the disease, highlighting its high mortality.

It is widely recognized that the pathogenesis of AHNE is caused by Clostridium perfringens type $\mathrm{C}$, an anaerobic gram-positive bacillus $(5,15)$. Clostridium perfringens type $\mathrm{C}$ can produce beta toxin, an oligomerizing, $35 \mathrm{kDa}$ pore forming cytopathic toxin that induces apoptosis and lysis $(16,17)$. The pores formed by beta toxin in the cell membrane allow the entry of $\mathrm{Ca}^{2+}, \mathrm{Na}^{+}$and $\mathrm{Cl}^{-}$into the cells and the efflux of $\mathrm{K}^{+}$, which leads to cell swelling and necrosis with sufficient potency to initiate the intestinal necrosis (18). Interestingly, diabetes mellitus which increases susceptibility to infection, and is associated with delayed gastric emptying and reduced intestinal mobility, has been reported as a risk factor for developing AHNE (14). Similarly, six of the ten patients reviewed in our study had a previous history of diabetes mellitus. Other risk factors for AHNE includes malnutrition, protein deficiency, sudden intake of proteinaceous meals, ingestion of Clostridium perfringens type $\mathrm{C}$ contaminated pig meat, and high dietary amounts of trypsin inhibitors $(15,16)$. Each of these risk factors contributes to beta toxin persistence in the gastrointestinal tract during Clostridium perfringens type $\mathrm{C}$ infection.

The main clinical symptoms of AHNE in the reported literature include diarrhea, hematochezia, abdominal pain, abdominal distention, nausea, vomiting and hypotensive shock. According to these clinical symptoms, AHNE can be classified into four types: type I acute toxic form; type II acute surgical form; type III subacute surgical form and type 


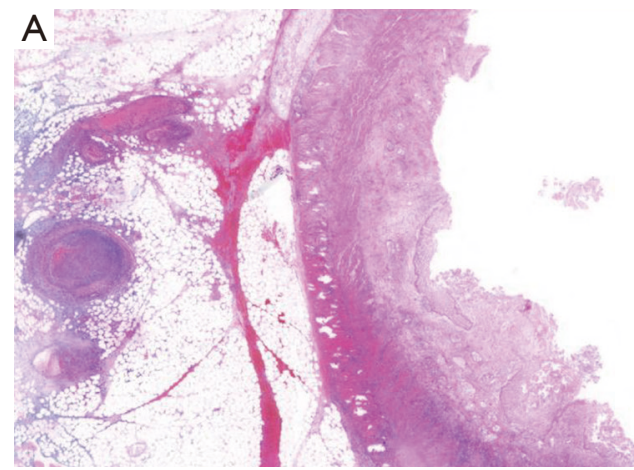

B
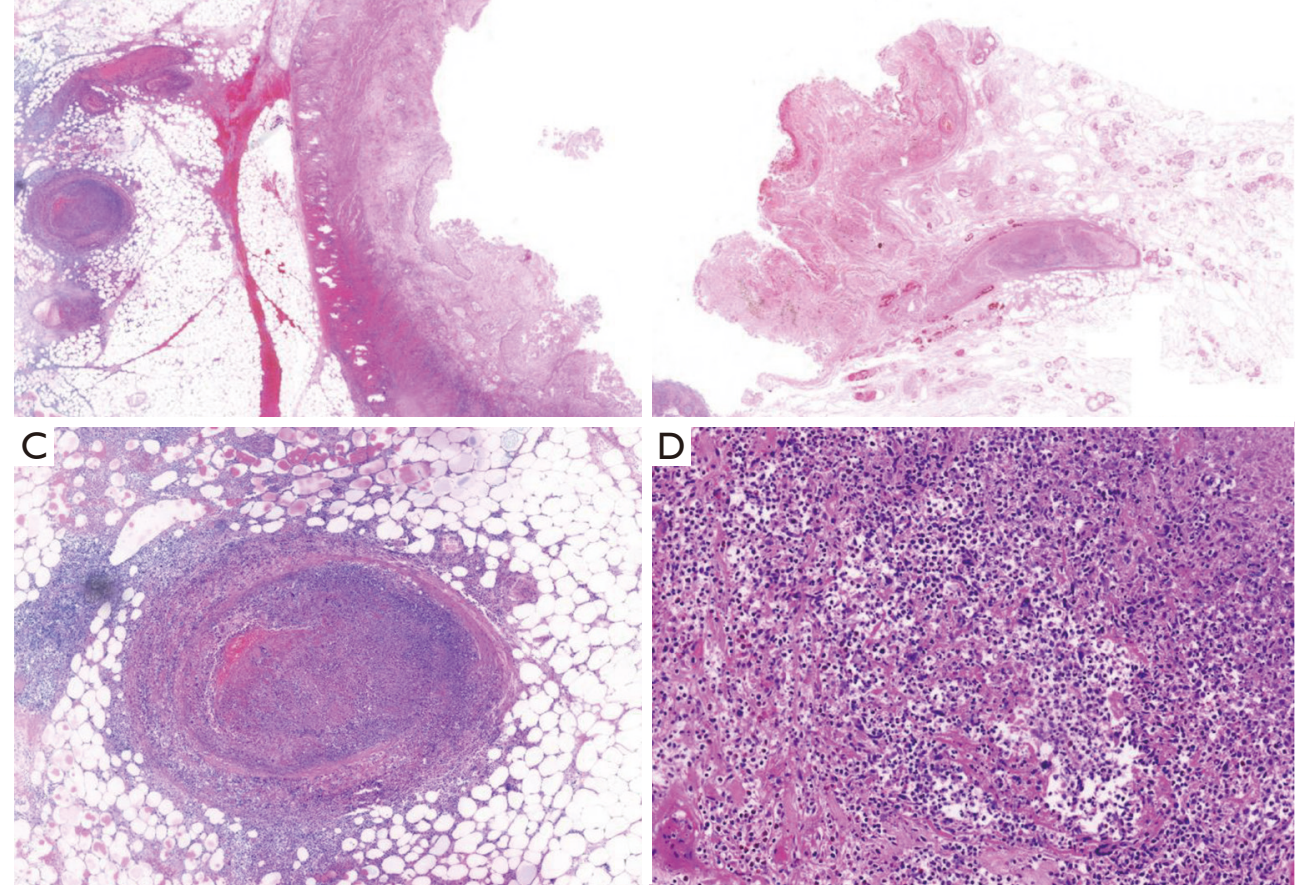

Figure 3 Pathology of resected bowel segments. (A) Hematoxylin-eosin staining revealed extensive necrosis of the intestinal mucosa with subserosal hemorrhage and fibrinoid vascular necrosis accompanied by endovascular thrombosis (magnification: $\times 100)$; (B) hematoxylineosin staining revealed full-thickness bowel-wall necrosis (magnification: $\times 100$ ); (C) hematoxylin-eosin staining revealed fibrinoid vascular necrosis accompanied by endovascular thrombosis (magnification: $\times 200$ ); (D) hematoxylin-eosin staining revealed local infiltration of neutrophils (magnification: $\times 200$ ).

IV mild or trivial form (5). During the course of the disease, a certain type can be the main clinical manifestation, but can also alternate, overlap or interchange.

Establishing a diagnosis of AHNE is made on the basis of the clinical manifestations and laboratory examination. In patients with a history of contaminated food, presenting with sudden progressively worsening abdominal pain, diarrhea, hematochezia, accompanied by hypotensive shock or ileus, AHNE should be highly suspected. On abdominal radiograph, dilated loops of small bowel with multiple air-fluid levels and gas in the bowel wall may be visible. Typical abdominal CT scan findings include segmental lesions of the small bowel, bowel wall thickening and edema as well as dilated bowel loops with or without diffuse peritonitis. In addition, pathological examination shows extensive segmental mucosal/submucosal and transmural necrosis with hemorrhage and pneumatosis $(11,12)$. The forementioned examinations further support the clinical diagnosis of AHNE while culture of Clostridium perfringens type $\mathrm{C}$ enables clear diagnosis of AHNE. The differential diagnosis of AHNE includes Crohn's disease, strangulated ileus, infectious colitis including amoebic colitis and bacillary dysentery.

Treatment involves a combination of medical therapy and timely surgical intervention. Principles of conservative management mainly include nil per os (NPO), gastrointestinal decompression, early fluid resuscitation, maintenance of water-electrolyte and acid-base balance, parenteral nutrition and somatostatin to inhibit mucosal secretions and reduce gastrointestinal hemorrhage. Early combination of empiric broad-spectrum antibiotics therapy including carbapenems, cephalosporins, fluoroquinolone and metronidazole is essential. Corticosteroids can reduce intoxication symptoms, inhibit the inflammatory response and improve the systemic response to stress. As for patients with severe sepsis and septic shock, short-term high-dose prednisolone can be injected intravenously for effective treatment. It was reported that 42,000 to 85,000 units of beta-antitoxin given intravenously can significantly reduce mortality from $43.9 \%$ to $18.7 \%$ (5). When active 


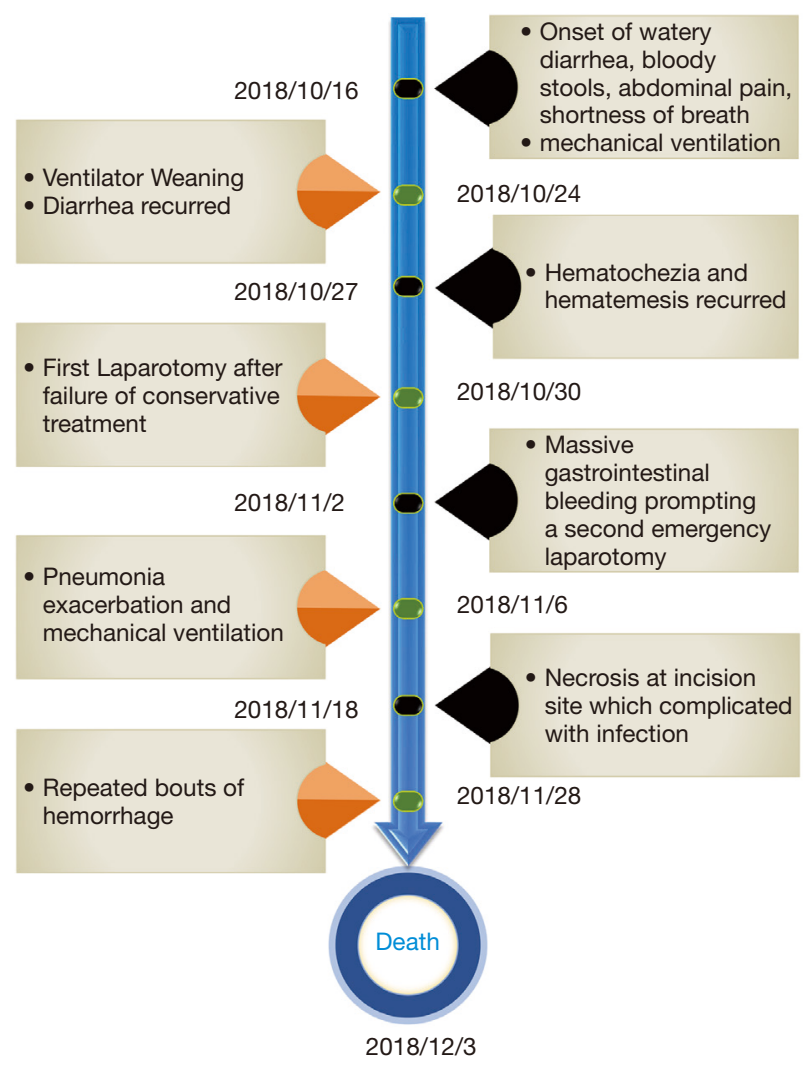

Figure 4 The timeline of the course of disease.

conservative treatment fails, timely surgical intervention is crucial. Absolute surgical indications include: (I) intestinal perforation; (II) severe intestinal necrosis with purulent or bloody effusion in abdominal cavity; (III) repeated massive gastrointestinal bleeding leading to haemorrhagic shock, despite aggressive fluid resuscitation; (IV) aggravation of mechanical or paralytic ileus.

Figure 5 shows a summary of the diagnosis and treatment of AHNE based on our review of current medical literature. In our case, the diagnosis of AHNE was a clinical one, supported by clinical and histopathological findings, without evidence of Clostridium perfringens type $\mathrm{C}$ on Gram stain. Subsequent testing for Clostridium perfringens type $\mathrm{C}$ beta toxin was negative while serological testing for antibodies to Clostridium perfringens type $\mathrm{C}$ was not available but is known to occur in $50 \%$ of patients with AHNE (10). Our case was highly challenging due to acute onset of clinical manifestations leading to respiratory failure, sepsis and multiple organ dysfunction syndrome. After initiating conservative treatment, the patient's condition remarkably improved despite presence of multiple comorbidities.
The patient unfortunately developed recurrent refractory gastrointestinal bleeding which was the leading cause of death despite of active medical treatment and timely surgical intervention.

\section{Conclusions}

In summary, AHNE is a life-threatening infectious disease with a high mortality rate, triggered by beta toxin produced by Clostridium perfringens type $\mathrm{C}$ under specific dietary conditions, characterized by extensive hemorrhagic, inflammatory, or ischemic necrosis that mainly affects the small bowel, with the main clinical manifestations including diarrhea, hematochezia, abdominal pain, abdominal distention, nausea, vomiting and hypotensive shock. Diagnosis is mainly based on the clinical features and auxiliary examination. A combination of medical therapy and timely surgical intervention are the main treatment methods of AHNE. To our knowledge, this is the first case report that attempts to summarize the pathogenesis, clinical characteristics, diagnostic methods, treatment and prognosis 


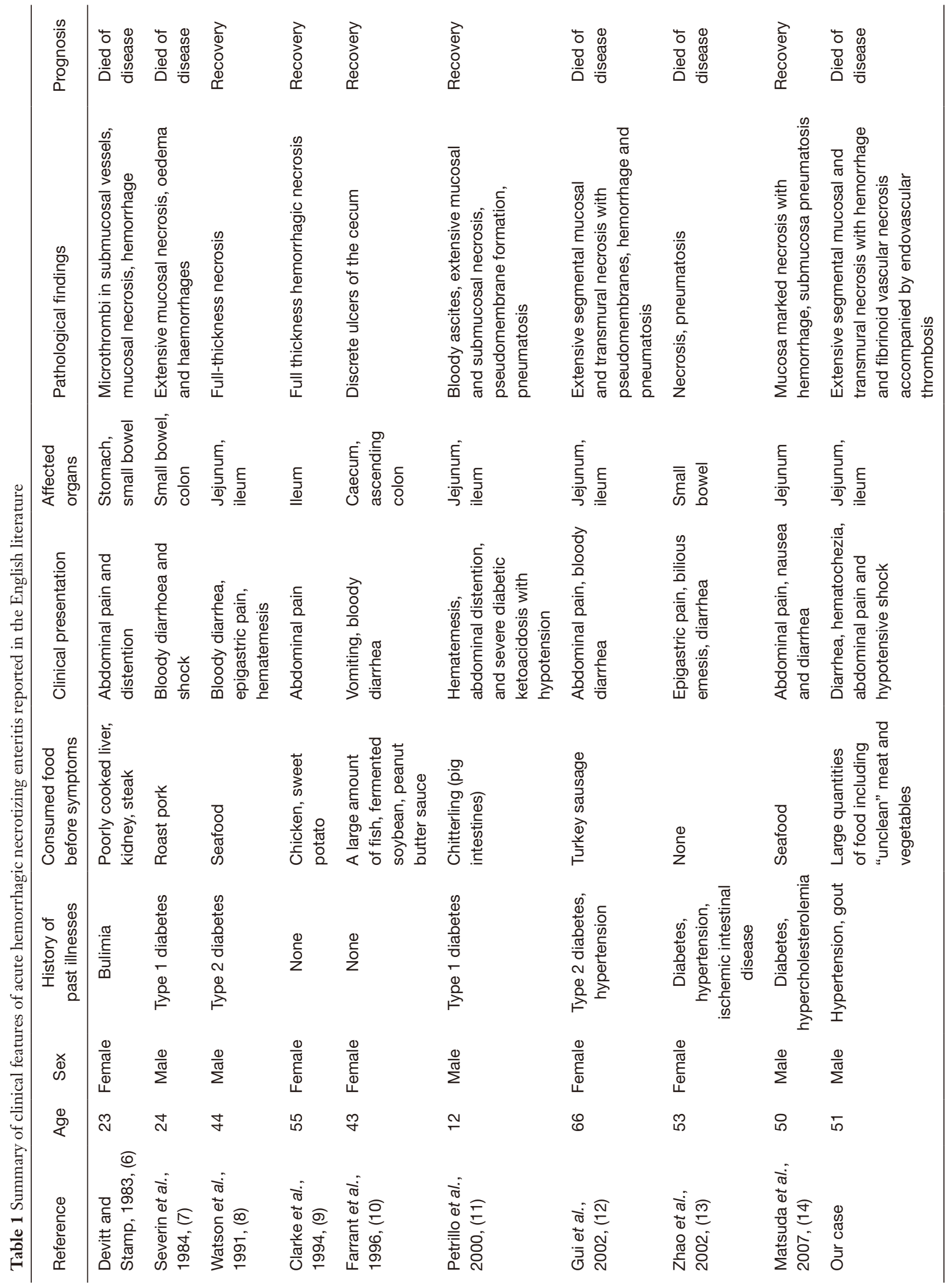




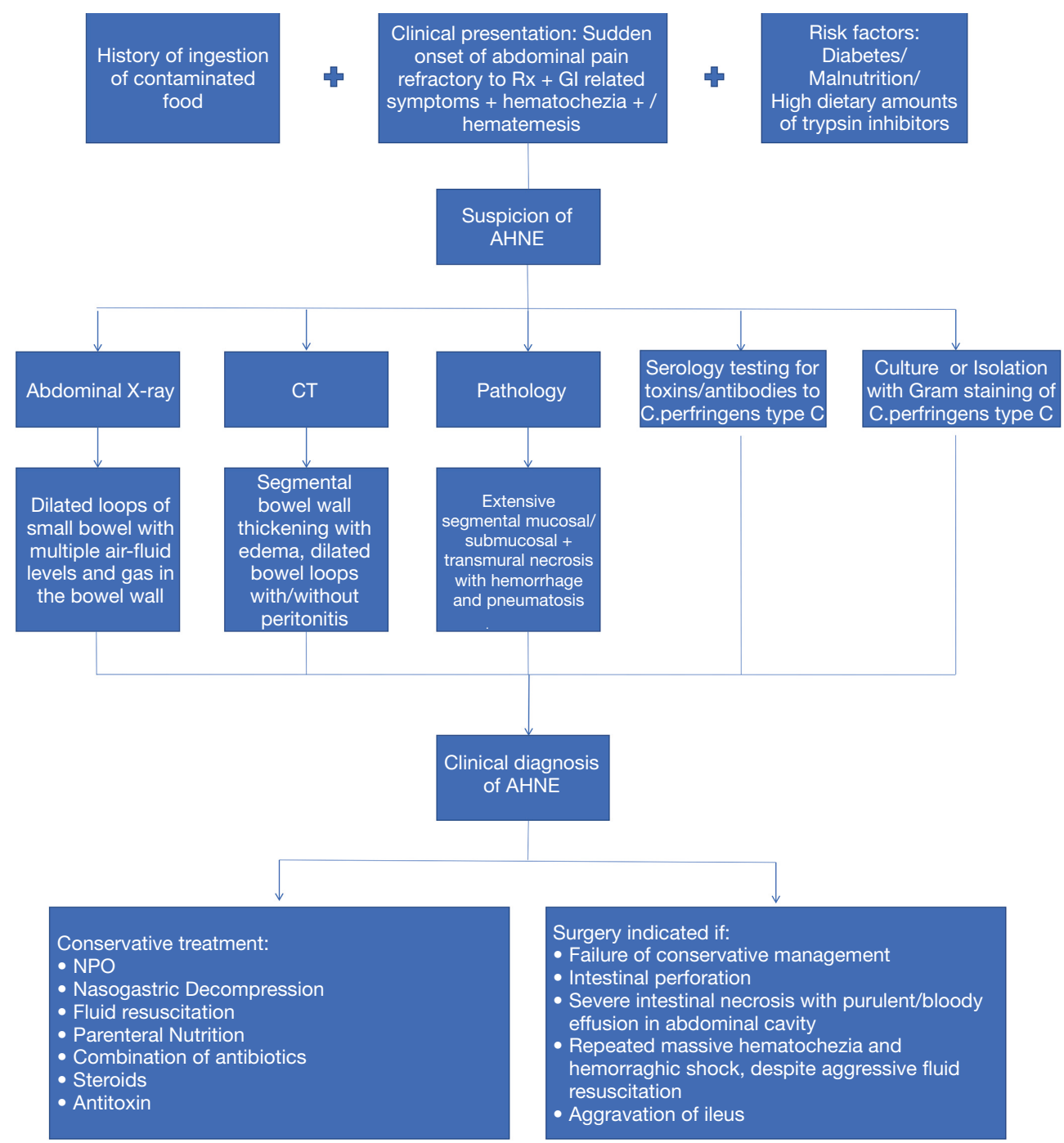

Figure 5 The flow chart of diagnosis and treatment of AHNE.

of AHNE based on the current literature. In order to reduce the mortality of this disease, emphasis should be laid on early recognition and timely surgical intervention in AHNE. In severe cases, death cannot be avoided despite adopting active supportive treatment and timely surgical intervention.

\section{Acknowledgments}

Funding: This work was supported by the National Natural Science Foundation for Young Scientists of China
(81800458).

\section{Footnote}

Reporting Checklist: The authors have completed the CARE reporting checklist. Available at http://dx.doi.org/10.21037/ apm-20-1131

Conflicts of Interest: All authors have completed the ICMJE uniform disclosure form (available at http://dx.doi. org/10.21037/apm-20-1131). The authors have no conflicts 
of interest to declare. No authors serve as a current Editorial Team member for this journal.

Ethical Statement: The authors are accountable for all aspects of the work in ensuring that questions related to the accuracy or integrity of any part of the work are appropriately investigated and resolved. All procedures performed in studies involving human participants were in accordance with the ethical standards of the institutional and/or national research committee(s) and with the Helsinki Declaration (as revised in 2013). The patient and his legal guardian provided informed written consent for publication of this study and any accompanying images during the treatment.

Open Access Statement: This is an Open Access article distributed in accordance with the Creative Commons Attribution-NonCommercial-NoDerivs 4.0 International License (CC BY-NC-ND 4.0), which permits the noncommercial replication and distribution of the article with the strict proviso that no changes or edits are made and the original work is properly cited (including links to both the formal publication through the relevant DOI and the license). See: https://creativecommons.org/licenses/by-nc-nd/4.0/.

\section{References}

1. Fick KA, Wolken AP. Necrotic jejunitis. Lancet 1949;1:519-21.

2. Zeissler J, Rassfeld-Sternberg L, Oakley CL, et al. Enteritis Necroticans due to Clostridium Welchii Type F. BMJ 1949;1:267-9.

3. Murrell TG, Roth L. Necrotizing jejunitis: a newly discovered disease in the highlands of New Guinea. Med J Aust 1963;50:61-9.

4. Murrell TG, Egerton JR, Rampling A, et al. The ecology and epidemiology of the pig-bel syndrome in man in New Guinea. J Hyg (Lond) 1966;64:375-96.

5. Murrell TG, Roth L, Egerton J, et al. Pig-bel: enteritis necroticans. A study in diagnosis and management. Lancet

Cite this article as: Zeng S, Tsia Hin Fong CJ, Li L, Liang Y, Liang Q, Wei B, Tao J. Acute hemorrhagic necrotizing enteritis: a case report and review of the literature. Ann Palliat Med 2021;10(5):5853-5861. doi: 10.21037/apm-20-1131
1966;1:217-22.

6. Devitt PG, Stamp GW. Acute clostridial enteritis--or pigbel? Gut 1983;24:678-9.

7. Severin WP, de la Fuente AA, Stringer MF. Clostridium perfringens type $\mathrm{C}$ causing necrotising enteritis. J Clin Pathol 1984;37:942-4.

8. Watson DA, Andrew JH, Banting S, et al. Pig-bel but no pig: enteritis necroticans acquired in Australia. Med J Aust 1991;155:47-50.

9. Clarke LE. Enteritis Necroticans With Midgut Necrosis Caused by Clostridium perfringens. Arch Surg 1994;129:557.

10. Farrant JM, Traill Z, Conlon C, et al. Pigbel-like syndrome in a vegetarian in Oxford. Gut 1996;39:336-7.

11. Petrillo TM, Beck-Sague CM, Songer JG, et al. Enteritis necroticans (pigbel) in a diabetic child. $\mathrm{N}$ Engl J Med 2000;342:1250-3.

12. Gui L, Subramony C, Fratkin J, et al. Fatal enteritis necroticans (pigbel) in a diabetic adult. Mod Pathol 2002;15:66-70.

13. Zhao W, Daroca PJ, Crawford BE. Clostridial enteritis necroticans versus secondary clostridial infection superimposed upon ischemic bowel disease. J La State Med Soc 2002;154:251-5.

14. Matsuda T, Okada Y, Inagi E, et al. Enteritis necroticans 'pigbel' in a Japanese diabetic adult. Pathol Int 2007;57:622-6.

15. Lawrence G, Walker PD. Pathogenesis of enteritis necroticans in Papula New Guinea. Lancet 1976;1:125-6.

16. Sayeed S, Uzal FA, Fisher DJ, et al. Beta toxin is essential for the intestinal virulence of Clostridium perfringens type C disease isolate $\mathrm{CN} 3685$ in a rabbit ileal loop model. Mol Microbiol 2008;67:15-30.

17. Uzal FA, McClane BA. Recent progress in understanding the pathogenesis of Clostridium perfringens type $\mathrm{C}$ infections. Vet. Microbiol 2011;153:37-43.

18. Uzal FA, Navarro MA, Li J, et al. Comparative pathogenesis of enteric clostridial infections in humans and animals. Anaerobe 2018;53:11-20. 